\title{
An Analysis of the Electromagnetic Environment of UHV-grade Transmission
}

\author{
Xie Lianke, Zhang Yong, Zang Yuwei, Ma Xingang, Zhang Guoying
}

State Grid Shandong Electric Power Research Institute, Shandong Jinan, 250002

Keywords: UHV; grade transmission; electromagnetic environment

\begin{abstract}
With the further implementation of the electricity-transmission project from the west to the east, the electromagnetic environment of the transmission project is closely related to the health of the people, the cost of the project, the national economic development and so on. Meanwhile, it is also a problem that needs to be fully considered in the process of raising the transmission voltage. This thesis analyzes the electromagnetic interference, noise, magnetic field presented in the transmission phenomenon of the AC UHV, the DC voltage of $800 \mathrm{KV}$ and even higher-grade voltage at home and abroad. And it puts forward relevant suggestions for improving electromagnetic environment, hoping to provide theoretical help for related workers in the future.
\end{abstract}

\section{Introduction}

In recent years, China is vigorously developing electricity-transmission project. In the process of electricity-transmission, higher-grade voltage transmission is one of the most important issues. Meanwhile, with the continuous development of the global economy and problems faced by the electric field in our country, the problem of the influence of electromagnetic environment of the transmission project is more and more obvious. And with the direct constraints of environmental project, the electromagnetic environment leads to the obvious increase of the structure, construction cost, and cost and so on of the transmission line. For this, it is of great practical significance to discuss the electromagnetic environment of UHV-grade transmission.

\section{Present Status of Related Research Abroad}

The electromagnetic environment in transmission project has already existed. Meanwhile, many foreign countries have carried out many researches and practices of electromagnetism for many years. In order to better solve the problem of the influence of electromagnetic environment of UHV transmission lines, the US, Italy, Japan and other countries have firstly established corresponding test base before taking new voltage grades and carried out a large number of experimental verifications. They design the transmission lines based on meeting economic condition, public recognition and other aspects ${ }^{[1]}$. The influence of electromagnetic environment of transmission project is mainly embodied in electromagnetic effect, radio interference, and audible noise and so on. In order to better meet the requirements of long-distance transmission, networking and so on, it is analyzed by using the technology of AC UHV transmission in the world. Meanwhile, many countries have made outstanding achievements in this field and some countries have already started to construct the transmission lines of AC UHV. Among them, the US is the first country to study UHV transmission and its research results in this area are more prominent.

\section{The Present Research Status of electromagnetic environment of UHV-grade transmission}

\subsection{Transmission Line}

In terms of audible noise, from the experience of research, analysis, design, construction and application, the radio interference of the lines of $750 \mathrm{KV}$ and below is controlled by design method of lines. The design of the lines can basically meet the requirement of radio interference limit. Meanwhile, for UHV lines, it is obvious that the control of the audible noise cannot be satisfied only with the help of the design of lines ${ }^{[2]}$. The research result of audible noise is more ideal. 
According to the experimental results, it can be summarized as the formula of analyzing computational experience and the methods of arranging additional sub-wire through the non-symmetric and multi-split conductor and the lower part of wire, conducting the aging treatment of insulated skin of sub-conductor, applying the tax-increase coating not affecting heat dissipation of the conductor. In terms of radio interference, the research on radio interference is mainly conducted under the recommendation of the Special Committee on Radio Interference. It gives the branching scheme about the experience of calculating the radio interference level of UHV transmission line and puts forward corresponding protect-distance scheme and many measures to reduce the radio interference according to the working characteristic and kind of wireless radio are. After comprehensively analyzing and considering, we need to improve in many aspects in the process of the construction of UHV transmission line. For example, we should control the radio interference by increasing the number of the division and the interphase distance, reducing the interface of conductor appropriately and other methods. In some places, we can also improve the radio interference limit appropriately to reduce the impact of interference on social operation ${ }^{[3]}$. In terms of electric field effect, the effect of the ecological effect of electric field can be divided into two kinds, short-term effect and long-term effect. The short-term effect is mainly to study people's direct feel of electric shock and electric field and the influence of the current for human body. The study of long-term effect is conducted mainly with the help of research methods of investigation and test. The effect of power frequency electromagnetic field on biological system, especially whether it will affect human health, remains to be studied. The research on ecological effects still needs further researching. Besides the voltage, the specific situation of the spatial field strength of the transmission line is also closely related to the arrangement of the wire. For this, it is necessary to adjust and optimize the height from the wire to the ground, the distance, the division size and so on.

\subsection{Substation}

There are mainly three types of UHV substations currently available, which are traditional outdoor substation, hybrid substation and gas-insulation GIS substation. Then it will take the gas-insulation GIS substation for example to discuss the influence of electromagnetic environment ${ }^{[4]}$.

In the 1970s, the electric enterprises in the developed countries of Europe and America establish the "digital grid" as the sally port for production and operation by using GIS technology, which improved the operation and management level of power grid and enhanced the competitiveness of electric enterprises themselves. In recent years, promoted by the policies of vigorous promotion for the process of electricity marketization, further construction and renovation of urban and rural power grid, improvement of information degree of electric industry and so on in China, the enterprises at all levels in our country have also begun to support and pay attention to the construction of transport and inspection system of GIS transmission lines of electric enterprises ${ }^{[5]}$. GIS transmission line has done something in the actual application and also is of a certain application value in terms of electromagnetic environment. Taking a company in China for example, the company, through technological innovation, taking the 3D GIS as basic platform and the third generation data-interaction system composed of WIFI+MESH as the communication basis, integrates the existed anti-break outside and remote control platform, and introduce online monitor, UAV inspection, handheld visual intelligent inspection and other application systems to make the channel of transmission wire 3D visualized and the site management more efficient. Meanwhile, with the help of the application case of the company, we sum up the methods of reducing electromagnetic effect based on GIS transmission line: 1. through the seamless connection of the $0.1 \mathrm{M}$ high-accuracy topography and terrain image of line channel and the GIS geographic information map of State Grid Corporation, achieve the 3D scene modeling of line corridor. Make the line equipment 3D-information. Improve the efficiency of operation and maintenance work of line operation and timely implement the precaution and control for electromagnetic environmental influence at the same time according to the situation of operation and maintenance work; 2 . The self-built data-interaction platform integrates a variety of advanced technology and support all kinds 
of wireless devices flexibly accessed. It can keep up with the development of information technology and provide technical support for the transformation of management idea. According to the implementation monitor of the electromagnetic effect, make implementation analysis of the data obtained from the monitor and reduce the electromagnetic environmental effect; 3 . The operation and maintenance management of transmission line change from the traditional labor-intensive patrol way to the information and 3D patrol, which improves the intellectualization level of power grid and the control ability of substation itself in electromagnetic environment at the same time.

\section{Suggestions for Improvement}

In the future, in order to better improve the problem of electromagnetic environment of UHV-grade transmission, the corresponding standards should be set up first. For the transmission line of AV UHV, audible noise level should be controlled within 52db at the location of 15m away from the side-phase wire. Meanwhile, the radio interference level 20m away from the side-phase wire should be controlled from 55 to $60 \mathrm{db}^{[6]}$. Meanwhile, take a variety of ways to control the power field strength of UHV transmission line: 1 . For the public active region, the field strength needs to be controlled within $5 \mathrm{KV} / \mathrm{m} ; 2$. When the line is across the road or public places, the field strength needs to be strictly controlled and should not be over $7 \mathrm{KV} / \mathrm{m}$. When it is across the farmland, it should be controlled within $10 \mathrm{KV} / \mathrm{m}$; 3 . For the less populated area, the field strength at the location where the line crossing the highway cannot be over $10 \mathrm{KV} / \mathrm{m}$. For the area of non-public active area or occasional person-passing area, the field strength can be relaxed to $15 \mathrm{KV} / \mathrm{m}$ in terms of control. For the $800 \mathrm{KV}$ an even higher voltage-grade DC transmission line, the index of electromagnetic environment can be controlled in about $500 \mathrm{KV}$, which means controlling the synthetic field in $30 \mathrm{KV} / \mathrm{m}$ and the density of particle flow in $100 \mathrm{nA} / \mathrm{m} 2$. Compared with other countries, China is of large population density and the line often is across or around the houses, so we should analyze construction investment foe transmission line when it refers to public health problems. Generally speaking, at the position $15 \mathrm{~m}$ away from the positive polarity wire, $50 \%$ of the audible noise level of all-weather should be controlled in the range of 45 to $50 \mathrm{db}$ and the radio interference level should be controlled according to the relevant regulations of China.

According to the research result and practical experience of transmission technology in developed countries and the actual conditions in our country, it should be targeted treated in the process of line construction according to the proportion of investment in different regions ${ }^{[7]}$. For this, we should constantly learn and summarize the research result of UHV transmission in various countries and choose long-span UHV test line of more suitable assumption and corona cage in our country according to the actual conditions of our country. Meanwhile, we should carry out a number of studies and guide the future work according to the research result. The specific contents of the study are mainly as follows: 1 . Environment impacts test. Compared with other countries, China is of the characteristics of large population density and serious environmental problems. In addition, regional environment is complex and climate change is prominent in China. Especially the high-altitude areas occupy $75 \%$ of China. These areas are the main location of hydropower resources and large fuel power in China, so they are also the main areas where is of the problem of corona discharge. For this, we need to analyze UHV transmission environment in terms of two aspects of theoretical research and experimental analysis according to the high altitude. Meanwhile, we can make clear about the size of wire structure and the minimum height away from ground with the help of corresponding tests. 2. Electromagnetic environment limit Test. The limit of electromagnetic environment of UHV transmission project in line with our actual situation and the limit must meet the requirements of the basic national conditions, economic cost and other aspects in China. 3. The calculation study of electromagnetic environment. Implement the collection, synthesis, integration, induction and analysis of the existed calculation methods and verify the experimental result with the method of comparison. Further discuss the noise limit of EHV and UHV transmission line. For the problem of radio interference, it can be calculated offline by the method of power frequency electric field and power frequency magnetic field. Study the calculation method of the synthesis the electric field and ion flow of DC transmission line; 4. Optimize and 
improve the environmental effect measures. Continually discuss and communicate the noise control measures for UHV transmission line and the ecological effect of electric field, the control method of the noise of UHV converter station, the most optimized structure, the shape and the grounding performances of DC grounding electrodes. Discuss the influence of the potential ascension of DC grounding electrodes and AC system. Meanwhile, make clear about the way to control the DC current of AC system and analyze electromagnetic influence of EHV and UHV transmission systematization on weak electric system, long-distance transmission system, and oil and gas pipeline and so on.

\section{Conclusions}

To sum up, with the continuous increase of voltage grade and the line transmission capacity, there must be a series of environmental problems. For this, we need to make improvement and adjustment in many aspects in the future. For example, carry out a number of studies, and improve adjust the transmission mode according to the actual situation of transmission line in China and reduce the negative impact of the electromagnetic environment to improve the transmission efficiency proposed in this paper.

\section{References}

[1] Liu Lina, Tao Jiaxiang, Zhang Yemao, etc. Actual Measurement Analysis of Electromagnetic Environment of Transmission Line of UHV Communication and Demonstration Project [J]. China Electric Power, 2017, 50(10): 46-51.

[2] Zhang Yemao, Wan Baoquan, Zhou Bing, etc. Statistical analysis of Long-term Test Data of Audible Noise of UHV Transmission Line [J]. Chinese Journal of Electrical Engineering, 2017, 37(20): 6136-6144.

[3] Xiao Dongping, Jiang Keru, Zhang Zhanong, etc. Optimization Method of Structure Layout of EHV/UHV Transmission Lines under the Constraint of Power Frequency Electromagnetic Environment [J]. Chinese Journal of Electrical Engineering, 2015, 35(9): 2333-2341.

[4] Liu Lei, Tian Feng, Li Min, etc. Electromagnetic Environment Prediction of High-altitude UHV DC Transmission Line under the Influence of Multiple Physical Factors [J]. South Grid Technology, 2016, 10(9): 43-48.

[5] Deng Jun, Xiao Yao, Chu Jinwei, etc. Simulation Calculation and Test Analysis of Synthetic Electric Field of $\pm 800 \mathrm{kV}$ DHV DC Transmission Line in Yunnan and Guangdong [J]. Electric Automation Equipment, 2015, 35(2): 138-144.

[6] Li Qian, Liao Minchuan, Wang Xuejun, etc. Study on Co-tower Electromagnetic Environment of Polar Wire and Grounding Electrode Line of $\pm 800 \mathrm{kV}$ UHV DC Project from the Northwest of Yunnan to Guangdong [J]. South Grid Technology, 2016, 10(9): 49-54.

[7] Zhang Yemao, Zhang Jiangong, Wuxiong, etc. Statistical Analysis of Long-term Test Data of Radio Interference of Transmission Line of UHV Test Demonstration Project [J]. High Voltage Technology, 2015, 41(11): 3708-3714. 